\title{
IMPLEMENTAÇÃO E AVALIAÇÃO DE ALGORITMO DE CONTROLE PREDITIVO EMBARCADO EM PLC
}

\author{
CARLOS A. A. LOPES JUNIOR ${ }^{1}$, ANTONIO A. R. COELHO ${ }^{1}$. \\ 1. Departamento de Automação e Sistemas, Universidade Federal de Santa Catarina \\ 88040900, Florianópolis, SC, BRASIL \\ E-mails: calopesjunior@das.ufsc.br, aarc@das.ufsc.br
}

\begin{abstract}
This paper discusses the application of an advanced control technique, called model-based predictive control (MPC), using a programmable logic controller (PLC) as a digital platform to derive the MPC control algorithm. To reduce the computational cost it is used a simplified approach of the GPC (Generalized Predictive Control) control law, assessed in processes with low-order transfer function. Experimental results with laboratory scale plants are realized to show the effectiveness of the control law, and the embedded predictive controller is evaluated in an application of speed control of hydraulic turbines using an approach denominated hardware in-the-loop.
\end{abstract}

Keywords_ Model-based predictive control, Programmable logic controller, Embedded algorithm, Hardware in-the-loop.

Resumo- Este trabalho aborda a aplicação de uma técnica de controle avançada, denominada controle preditivo baseado em modelo, utilizando um controlador lógico programável (PLC) como plataforma de desenvolvimento deste algoritmo de controle. Visando uma redução do custo computacional é utilizada uma abordagem simplificada do método GPC - Generalized Predictive Control, válido para processos modelados por funções de transferência de ordem reduzida. Para mostrar a eficácia da técnica são realizados ensaios experimentais com um planta em escala de laboratório e uma simulação hardware in-the-loop utilizando o controlador embarcado no PLC como um regulador de velocidade de turbinas hidráulicas.

Palavras-chave— Controle preditivo baseado em modelo, Controlador lógico programável, Algoritmo embarcado, Hardware in-the-loop.

\section{Introdução}

O termo controle preditivo baseado em modelo (MPC) não se refere a apenas um algoritmo de controle específico, corresponde a uma classe de controladores que possui um modelo dinâmico do processo programado dentro do controlador, onde a função do modelo é fazer a predição do comportamento futuro do processo. Estes controladores são os mais utilizados, na indústria, dentre as técnicas de controle avançado existentes, segundo Haber (2011). A técnica de controle preditivo utilizada nesta dissertação é denominada Generalized Predictive Control (GPC)

O controle preditivo generalizado, método proposto por Clarke et al. (1987), é um representante da família MPC com popularidade na academia e indústria. O algoritmo de controle GPC pode ser abordado em duas partes: na primeira calcula-se um preditor ótimo para o cálculo da saída esperada, como função de controles passados e do estado atual do processo, na segunda encontra-se a lei de controle que minimiza uma função custo, que tipicamente combina o erro da saída e o esforço do controlador numa formulação única (Camacho e Bordons, 2007).

Para o cálculo e implementação dos parâmetros do GPC existe uma certa complexidade matemática, o que pode inviabilizá-lo em determinadas implementações práticas. Entretanto, a necessidade da elaboração de algoritmos de controle preditivo simples e eficientes visando a programação em plataformas do tipo PLC (Programmable Logic Controller), DSP (Digital Signal Processor), FPGA (Field-
Programmable Gate Array) ou em outros aplicativos microcontrolados (que são destaques na indústria) é uma necessidade em chão de fábrica e é evidente nos dias atuais (Cooper, 2004).

Alguns trabalhos publicados propõem métodos de transpor as limitações de processamento e capacidade de memória destes dispositivos. Besch et al. (2009) propõe uma forma de inserir ao PLC a lei de controle de um GPC escalonado de acordo com o ponto de operação, por meio da programação $L a$ dder. Para validação da implementação do GPC foi desenvolvida em Matlab® uma simulação de um planta de controle de $\mathrm{pH}$. Em Rohman e Sirius (2011) um MPC utilizando um algoritmo de programação quadrática foi implementado em um PLC da Mitsubishi. Para mostrar sua eficácia, o aplicativo foi utilizado para controlar a velocidade de um motor CC e comparado com um PI com anti-windup. Palomo e Rossiter (2011) apresentaram um algoritmo de controle preditivo capar de lidar com as restrições de recurso de um PLC, para isto foi desenvolvida uma abordagem paramétrica onde são aceitos casos subótimos. Apesar da sub-otimalidade, o algoritmo manteve garantia de estabilidade no caso nominal.

Visando uma redução do custo computacional do algoritmo MPC, este artigo utiliza uma implementação simplificada do método GPC proposta por $\mathrm{Ca}$ macho e Bordons (1998). O projeto é válido para processos que podem ser modelados por funções de transferência de ordem reduzida. Esta proposta foi denominada de Controlador Preditivo de Ordem Mínima (CPOM) e baseia-se no fato de que uma grande quantidade de processos industriais podem ser descritos por um pequeno número de parâmetros, modelos 
FOPDT (First Order Plus Dead-Time), e um conjunto de funções, simples, podem ser desenvolvidas para o cálculo dos parâmetros de sintonia deste controlador.

\section{Controlador preditivo de ordem mínima}

Grande parte dos processos na indústria quando se considera pequenas variações em torno do ponto de operação podem ser descritos por um modelo linear de ordem elevada. A razão para isto é que a maioria dos processos industriais é composta por muitos elementos dinâmicos, muitas vezes de primeira ordem, sendo o modelo completo de ordem igual ao número de elementos. Estes modelos de ordem elevada são difíceis de utilizar para fins de controle, mas é possível aproximar o comportamento de tais processos por um modelo linear com um ganho estático, $K_{p}$, uma constante de tempo, $\tau$, e um atraso de transporte, $\theta$ (Camacho e Bordons, 2007).

Neste sentido, um grande número de plantas industriais pode ser aproximadamente modelado por

$$
G_{m}(s)=\frac{K_{p}}{\tau s+1} e^{-\theta s}
$$

Quando o atraso de transporte $\theta$ é um múltiplo inteiro do período de amostragem $T_{s}\left(\theta=d T_{s}\right)$, onde $d$ é o atraso de transporte discreto, a função de transferência discreta correspondente a Equação (1) é dada por

$$
G(z)=\frac{b_{0} z^{-1}}{1+a_{1} z^{-1}} z^{-d}
$$

A partir da Equação (2) e de um modelo linear discreto CARIMA (Controlled Auto-Regressive and Integrated Moving-Average) é possível obter o modelo interno de predição para o CPOM

$$
\begin{aligned}
& \hat{y}(t+d+j / t)=\left(1+a_{1}\right) \hat{y}(t+d+j-1 / t) \\
& -a_{1} \hat{y}(t+d+j-2 / t)+b_{0} \Delta u(t+j-1)
\end{aligned}
$$

os polinômios ruído foram escolhidos unitários.

O algoritmo do GPC consiste na aplicação de uma sequência de controle que minimiza a seguinte função custo:

$$
\begin{aligned}
J=E & \left(\sum_{j=N_{1}}^{N_{y}}[\hat{y}(t+j / t)-w(t+j)]^{2}+\right. \\
& \left.+\sum_{j=1}^{N_{u}} \lambda(j)[\Delta u(t+j-1)]^{2}\right)
\end{aligned}
$$

onde $\mathrm{E}\{\cdot\}$ é o operador esperança matemática, $N_{1}$ e $N_{\mathrm{y}}$ são os horizontes mínimo e máximo de saída, $N_{\mathrm{u}}$ é o horizonte de controle e $\lambda$ é o fator de ponderação do sinal de controle, sem perda de generalidade fazse $N_{1}=d+1$ e $N_{\mathrm{y}}=d+N$, sendo $N$ o horizonte de predição, e para simplificação da análise $N_{\mathrm{u}}=N$ (Camacho e Bordons, 2007).

Se a Equação (3) for aplicada recursivamente para $j=1,2, \ldots, N$ tem-se a equação matricial

$$
\hat{\mathbf{y}}=\mathbf{G u}+\mathbf{F} \mathbf{y}^{\prime}
$$

onde as matrizes $\mathbf{G}$ e $\mathbf{F}$ são constantes de dimensão $N$ $\times N$ e $N \times 2$, respectivamente, e os vetores $\hat{\mathbf{y}}=\left[\begin{array}{llll}\hat{y}(t+d+1) & \hat{y}(t+d+2) \ldots & \hat{y}(t+d+N)\end{array}\right], \quad \mathbf{u}=$ $[\Delta u(t) \Delta u(t+1) \ldots \Delta u(t+N-1)]^{T}$ e $\mathbf{y}^{\prime}=[\hat{y}(t+d / t)$ $\hat{y}(t+d-1 / t)]^{T}$.

Se $\hat{\mathbf{y}}$ é substituído na função custo (4) é uma função de $\mathbf{y}^{\prime}, \mathbf{u}$ e da sequência de referência futura $\mathbf{w}$. Minimizando $J$ com relação a u tem-se

$$
\mathbf{M u}=\mathbf{P y}{ }^{\prime}+\mathbf{R w}
$$

onde $\mathbf{M}=\mathbf{G}^{\mathbf{T}} \mathbf{G}+\lambda \mathbf{I}, \mathbf{R}=\mathbf{G}^{\mathbf{T}}$ são matrizes de dimensão $N \times N$ e $\mathbf{P}=\mathbf{- G}^{\mathbf{T}} \mathbf{F}$ de dimensão $N \times 2$. Fazendo $q$ a primeira linha da matriz $\mathbf{M}^{-1}$, então o incremento de controle é dado por

$$
\Delta u(t)=q \mathbf{P} \mathbf{y}^{\prime}+q \mathbf{R} \mathbf{w}
$$

Assumindo que a sequência de referência pode ser escrita como $\mathbf{w}=\left[\begin{array}{lll}1 & \ldots & 1\end{array}\right] y_{r}(t)$, e o incremento de controle é calculado através de

$$
\Delta u(t)=l_{y 1} \hat{y}(t+d / t)+l_{y 2} \hat{y}(t+d-1 / t)+l_{r 1} y_{r}(t)
$$

onde $q \mathbf{P}=\left[\begin{array}{ll}l_{y 1} & l_{y 2}\end{array}\right]$ e $l_{r 1} y_{r}(t)=q \mathbf{R w}$. Os coeficientes $l_{y 1}, l_{y 2}$ e $l_{r 1}$ são função de $a_{1}, b_{0}$ e $\lambda(j)$. Se o GPC é concebido tendo um ganho estático unitário, os coeficientes da Equação (8) só dependem de $\lambda(j)$, que é considerado constante e do polo do modelo estimado (Camacho e Bordons, 1998).

Esta metodologia padrão para o cálculo dos parâmetros do controlador, realizando operações com matrizes, que por vezes são de ordem elevada, pode ser inconcebível para algumas aplicações em tempo real com hardware limitado. A proposta de Camacho e Bordons (1998) foi pré-calcular esses parâmetros, para uma faixa de polos (0.5 a 0.95) e $\lambda$ e encontrar funções que aproximassem os valores de $l_{y 1}, l_{y 2}$ e $l_{r 1}$, as funções aproximadas para cálculo desses parâmetros são: 


$$
\begin{aligned}
& l_{y i}=k_{1 i}+k_{2 i} \frac{a}{k_{3 i}-a} \quad i=1,2 \\
& l_{r 1}=-l_{y 1}-l_{y 2}
\end{aligned}
$$

Os subcoeficientes $k_{j i}$ podem ser calculados por um conjunto de funções que foram obtidas fazendo $\lambda$ variar de 0.3 a 1.3 e um horizonte de predição fixo $N$ $=15$. Logo, os subcoeficientes podem ser calculados pelas seguintes funções:

$k_{11}=-\exp \left(0.3598-0.9127 \lambda+0.3165 \lambda^{2}\right)$

$k_{21}=-\exp \left(0.0875-1.2309 \lambda+0.5086 \lambda^{2}\right)$

$k_{31}=1.05$

$k_{12}=-\exp (-1.7383-0.40403 \lambda)$

$k_{22}=-\exp \left(-0.32157-0.81926 \lambda+0.3109 \lambda^{2}\right)$

$k_{32}=1.045$

Assim pode-se pensar na calibração do controlador preditivo de ordem mínima, em relação ao fator de penalização do controle $\lambda$, proporcionando respostas variando em modos dinâmicos conservativo, moderado e agressivo.

Para dar uma característica antecipativa à saída do sistema é utilizada uma técnica proposta por Moradi et al., (2001), onde uma nova referência é calculada através de

$$
\begin{aligned}
r(1)= & \sum_{j=1}^{N_{y}} y_{r}(j) / N_{y} \\
r(t)= & r(t-1)+\frac{y_{r}\left(t+N_{y}-1\right)}{N_{y}} \\
& -\frac{y_{r}(t-1)}{N_{y}}, \quad t=2, \ldots, n
\end{aligned}
$$

onde $n$ equivale ao número total de iterações. Podese dizer então que a nova referência gerada $r(t)$ corresponde a média dos valores da referência $y_{r}(t)$ nos próximos $N_{y}$ passos.

\section{Implementação do CPOM}

Uma vez que o fator de ponderação foi escolhido, os valores $k_{j i}, l_{y 1}, l_{y 2}$ e $l_{r 1}$ podem ser facilmente calculados por (10) e (9). Na Tabela 1 pode-se observar o algoritmo de controle a ser implementado.

Este algoritmo foi avaliado frente ao GPC padrão através de simulações numéricas em Matlab®. Foram utilizados dois tipos de plantas bastante comuns em processos industriais, uma planta com múltiplos polos iguais e outra com zero de fase nãomínima.
Tabela 1. Algoritmo CPOM.

\begin{tabular}{l}
\hline \multicolumn{1}{c}{ CPOM } \\
\hline 1 - Identificação off-line do modelo FOPDT; \\
2 - Escolha $\lambda$; \\
3 - Calcule os valores de $k_{j i}$ pela equação (10); \\
4 - Calcule os parâmetros do controlar por (9); \\
5 - Leia a variável do processo, $y(t) ;$ \\
6 - Calcule as predições da saída através de (3); \\
7 - Calcule o incremento de controle; \\
8 - Divida o sinal de controle pelo ganho estático; \\
9 - Retorne ao passo 5. \\
\hline
\end{tabular}

Os controladores foram avaliados pelos seguintes indicadores de desempenho: ITAE (Integral of Time multiplied by Absolute Error) para o comportamento servo, IAE (Integral of Absolute Error) e TVC (Total Variation of Control), e os indicadores de robustez que utilizam as funções de sensibilidade e sensibilidade complementar, encontrados em Seborg et al. (2010).

Os indicadores de desempenho foram melhores para o GPC padrão, entretanto com uma pequena diferença de aproximadamente $4.5 \%, 7.7 \%$ e $11.4 \%$ para ITAE, IAE e TVC, respectivamente. Os indicadores de robustez permaneceram dentro da faixa sugerida como guideline, ou seja, tiveram um bom compromisso entre desempenho e robustez.

Baseando-se nos índices calculados para estes estudos de caso tem-se que o CPOM teve um desempenho satisfatório e muito próximo do apresentado pelo GPC padrão, sob a mesma sintonia. Mais detalhes desta análise podem ser encontrados em Lopes Júnior (2013).

Após esta avaliação, o algoritmo do CPOM foi embarcado em um controlador lógico programável fabricado pela WEG Equipamentos Elétricos S. A., o PLC300. Este é um robusto controlador lógico programável que concentra as funcionalidades de PLC e IHM (Interface Homem Máquina), compacto, configurável e de baixo custo, é bastante atrativo para as necessidades dinâmicas no controle de plantas industriais.

Para a implementação do bloco CPOM no PLC300 foi definida a utilização da linguagem C. Entre as motivações para a escolha está o fato de ser uma linguagem simples que permite trabalhar com funções matemáticas, tipos de dados simples, acesso direto à memória, e o interesse por parte da empresa parceira, WEG Automação.

A partir do código em $\mathrm{C}$ do CPOM foi gerado o seguinte bloco para o controlador lógico programável, Figura 1, este pode ser utilizado no ambiente de programação em Ladder do PLC300. 


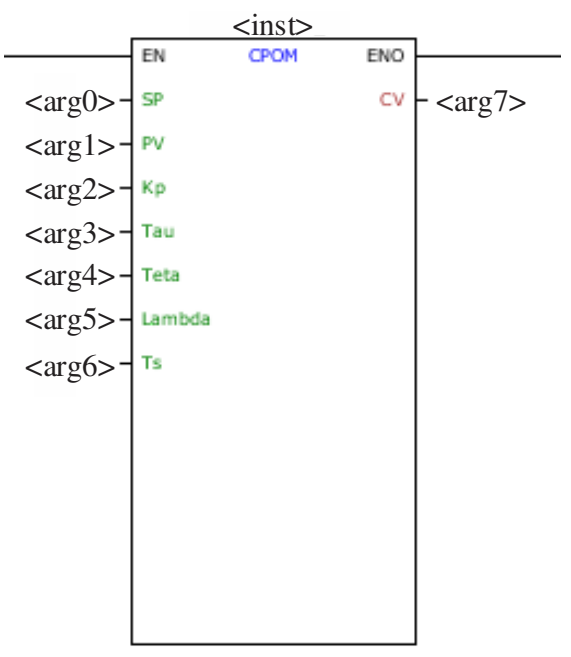

Figura 1. Bloco CPOM desenvolvido para o PLC300.

As entradas e saída do bloco são:

- $\quad \mathrm{SP}$ - Set point, é a referência para a variável do processo;

- $\quad$ PV - Variável do processo, é a realimentação da saída do processo;

- $\quad \mathrm{K}_{\mathrm{p}}$, Tau e Teta - São os parâmetros do modelo FOPDT do processo, são utilizados para predição da saída da planta;

- Lambda - É o fator de ponderação da variável de controle;

- $\mathrm{T}_{\mathrm{s}}$ - Período de amostragem do controlador;

- CV - Variável de controle, é a saída do controlador que deve atuar na entrada da planta.

Na próxima seção são tratados os resultados dos ensaios experimentais e Hardware In-the-Loop (HIL) realizados utilizando o $\mathrm{CPOM}$ embarcado no PLC300.

\section{Resultados}

\subsection{Simulação experimental}

Para mostrar a eficácia do CPOM embarcado no PLC300 foi realizado um ensaio com uma planta de nível em escala de bancada de laboratório.

A planta conta com dois tanques de armazenamento, um dos tanques é utilizado apenas como reservatório, no outro tanque é realizado o controle de nível. Na Figura 2 tem-se a imagem do processo utilizado nos ensaios.

Para aplicação do CPOM na planta de nível é necessário o modelo de primeira ordem com atraso de transporte. A identificação deste modelo FOPDT foi realizada através do método de Sundaresan apresentado em Coelho e Coelho (2004). O modelo FOPDT que caracteriza a planta de nível é apresentado em (12).

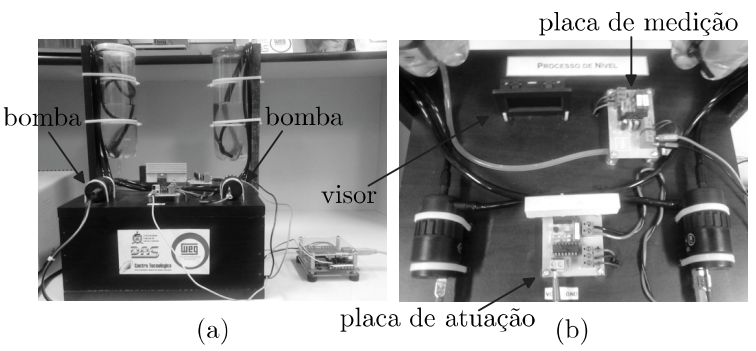

Figura 2. Planta de nível em escala reduzida. (a) Vista frontal. (b) Vista superior.

$G_{m}(s)=\frac{2.395}{79.365 s+1} e^{-1.165 s}$

Para a simulação foi utilizado um período de amostragem de $T_{s}=0.2 \mathrm{~s}$, e a penalização da variável de controle ajustada através de tentativa e erro, sendo $\lambda=1$. O ensaio teve três mudanças de referência: 1 , 2.5 e 4, a Figura(3) exibe a resposta do sistema e a variável de controle calculada pelo PLC300.
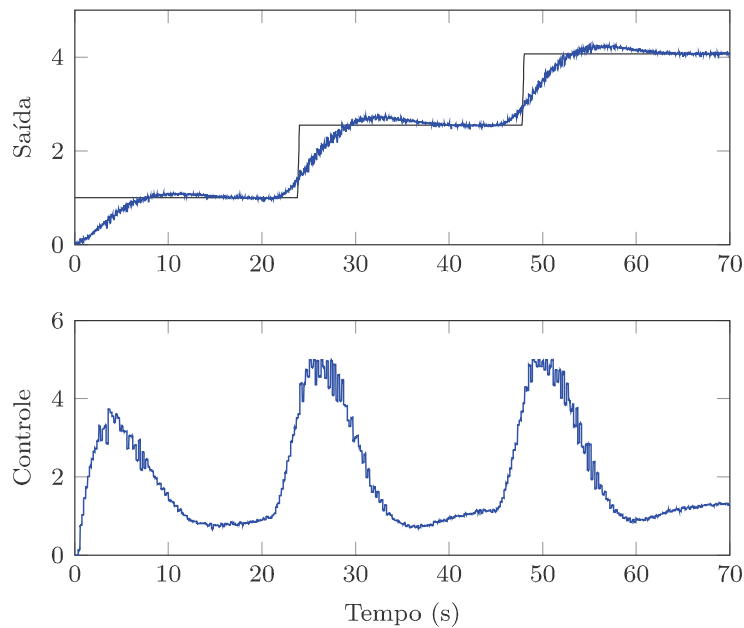

Figura 3. Seguimento de referência na planta de nível.

Percebe-se que a saída do sistema tem um pequeno sobressinal e depois converge para a referência desejada. Observa, ainda, na variável de controle uma pequena saturação imposta devido às limitações de entrada da planta de nível, entretanto esta saturação não compromete o desempenho do sistema.

\subsection{Ensaio Hardware In-the-Loop}

Adicionalmente, o controlador preditivo embarcado no PLC é avaliado numa aplicação como regulador de velocidade de turbinas hidráulicas, para tal, é utilizada uma abordagem denominada ensaios Hardware In-the-Loop. A função de regulação de turbinas hidráulicas para o PLC300 está em linha com o portfólio da WEG de soluções para pequenas centrais hidrelétricas.

A simulação HIL é uma técnica para desenvolvimento e testes de sistemas complexos. Estes ensaios são caracterizados pela operação em conjunto de componentes reais com outros simulados em tempo 
real, no caso, o hardware é o controlador (PLC300) e o software é uma simulação do processo.

O modelo do sistema de geração hidrelétrica utilizado nas simulações é dividido em três partes. Todas as grandezas com o sobrescrito “_” estão em normalizadas (pu).

Dinâmica dos posicionadores (Filho, 2011), Equação (13)

$$
\frac{\bar{g}}{C V}=\frac{1}{T_{g} T_{p} s^{2}+T_{g} s+1}
$$

onde $\bar{g}$ é a abertura real do distribuidor, $T_{p}$ é a constante de tempo resultante do servomotor piloto e válvula proporcional, $T_{g}$ é a constante de tempo resultante da válvula proporcional e distribuidor.

Dinâmica da turbina (não-linear) (Filho, 2011), Equações (14),

$$
\begin{aligned}
& \bar{G}=A_{t} \bar{g} \\
& \bar{H}=\left(\frac{\bar{U}}{\bar{G}}\right)^{2} \\
& \bar{U}=\frac{1}{T_{w} s}\left(\bar{H}_{0}-\bar{H}\right) \\
& \bar{P}_{m}=\bar{H}\left(\bar{U}-\bar{U}_{n l}\right) \\
& \bar{T}_{m}=\bar{P}_{r} \frac{\bar{P}_{m}}{\bar{\omega}}
\end{aligned}
$$

aqui, $\bar{G}$ é a abertura ideal do distribuidor, $\bar{U}$ é a velocidade da água na turbina, $\bar{U}_{n l}$ é a velocidade da água com turbina a vazio, $\bar{H}$ é a queda hidráulica medida do nível da turbina até o lago, $\bar{H}_{0}$ é a queda hidráulica inicial, $\bar{P}_{m}$ é a potência mecânica desenvolvida pela turbina, $\bar{\omega}$ é a velocidade do conjunto turbina-gerador, $\bar{T}_{m}$ é o torque mecânico desenvolvido pela turbina, $A_{t}$ é o ganho da turbina hidráulica, $T_{w}$ é o tempo de partida da água e $\bar{P}_{r}$ uma mudança de base para acoplar as grandezas da turbina com as do sistema de geração.

Dinâmica de rotação e carga (Filho, 2011), dada por (15),

$$
\bar{\omega}=\frac{1}{2 H_{g} s}\left(\bar{T}_{m}-\bar{T}_{e}-D \bar{\omega}\right)
$$

onde $\bar{T}_{e}$ é o torque eletromagnético, $H_{g}$ é a constante de inércia e $D$ é a constante de amortecimento para cargas isoladas.

Através da combinação dessas das Equações (13), (14) e (15) foi possível realizar um ensaio para identificação do modelo FOPDT para a planta. O método utilizado foi Sundaresan e Krishnaswamy, o mesmo aplicado na planta de nível. Este modelo é observado em (16), para sintonia do CPOM fez-se $\lambda$ $=0.5$, e para a simulação foi escolhido um $T_{s}=1.2 \mathrm{~s}$.

$$
G_{m}(s)=\frac{16.36}{139.39 s+1} e^{-6.6 s}
$$

O primeiro ensaio realizado foi de partida da máquina que ocorre em malha aberta. Na técnica utilizada a abertura do distribuidor é setada primeiramente em $30 \%$ até a velocidade de rotação da máquina alcançar $80 \%$ do seu valor nominal, a partir deste instante a abertura é limitada em $25 \%$ até atingir a velocidade nominal, neste momento o regulador de velocidade é habilitado e o distribuidor é liberado. $\mathrm{Na}$ Figura 4 é apresentado o ensaio de partida da máquina, que ocorre entre 0 e aproximadamente $42 \mathrm{~s}$, e então CPOM é habilitado. É possível observar um pequeno offset em relação à referência, isso ocorre devido a uma atenuação nos sinais de saída e entrada da placa de aquisição de dados, utilizada como interface de I/O entre o PLC e a simulação da planta.

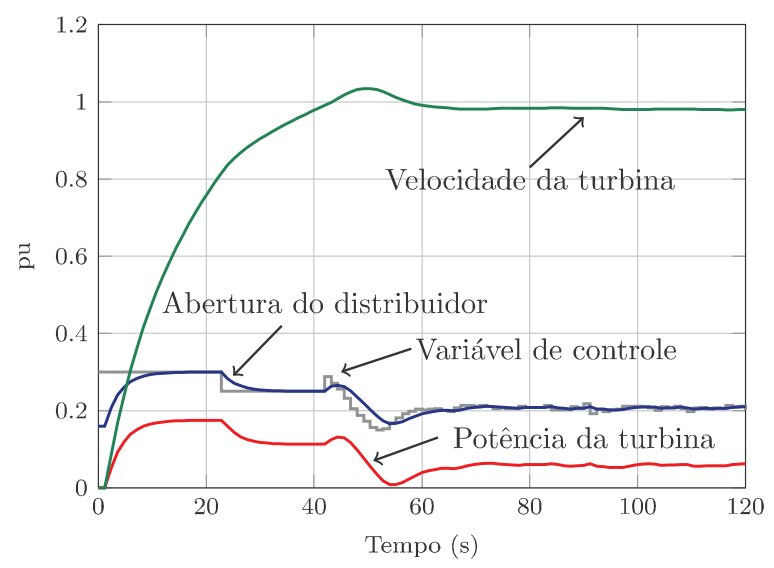

Figura 4. Seguimento de referência na planta de nível.

Como segundo ensaio, é realizado um teste de segmento de referência. A mudança aplicada é um degrau de amplitude 0.03 pu. Este ensaio é apresentado na Figura 5.
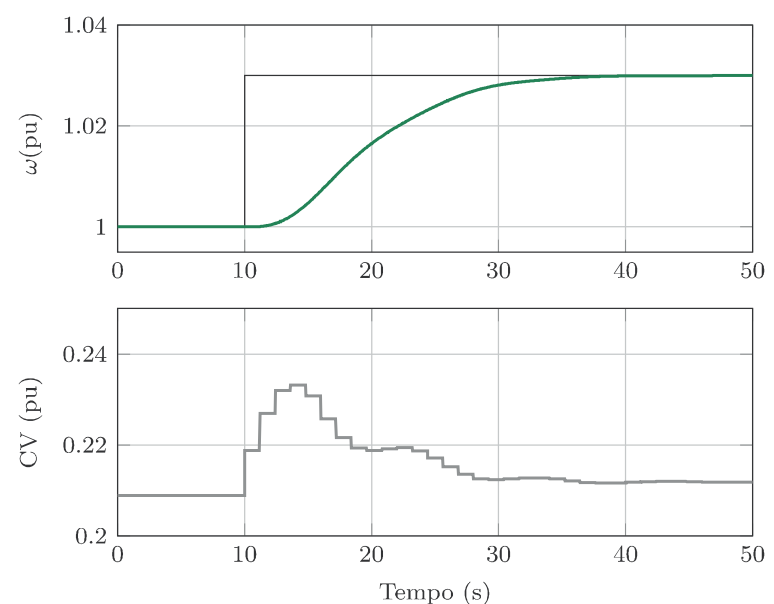
Figura 5. Seguimento de referência para um degrau de 0.03 pu de velocidade.

É possível observar um que o tempo de subida de aproximadamente $18 \mathrm{~s}$ e sem sobressinal.

Como terceiro ensaio fez-se um teste de variação de carga. A Figura 6 ilustra a resposta de sistema para perturbação de carga de $12 \%$ da potência nominal. Analisando os gráficos percebe-se que o CPOM rejeita a perturbação em aproximadamente $15 \mathrm{~s}$, ou seja, aumenta a geração de potência de forma a atender toda a carga inserida no sistema.
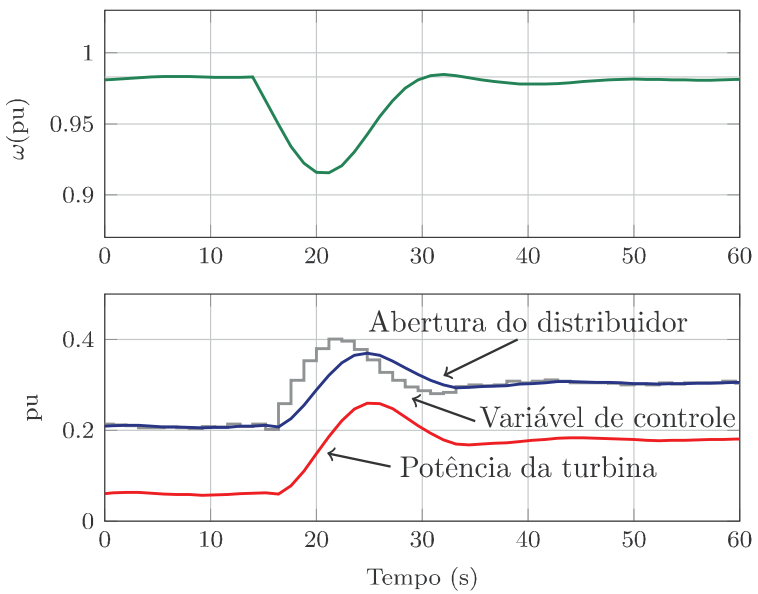

Figura 6. Rejeição de perturbação de carga de $12 \%$.

O CPOM mostrou ser um candidato em potencial para utilização como regulador da velocidade de turbinas hidráulicas, entretanto estudos complementares ainda devem ser realizados antes de sua aplicação prática final.

Estes ensaios mostraram a aplicabilidade do controlador preditivo de ordem mínima, que apesar de utilizar um modelo de ordem reduzida no projeto, na predição das saídas futuras e ter os parâmetros do controlador calculados através de funções aproximadas, teve um desempenho satisfatório controlando plantas não-lineares e de dinâmicas complexas.

\section{Conclusões}

Neste trabalho foi avaliada a implementação de uma técnica de controle preditivo embarcada em controladores lógicos programáveis. Uma das principais limitações do método aplicado é na escolha do fator de ponderação do controle, que deve ser escolhido no intervalo de 0.3 a 1.3. As diferenças e limitações apresentadas são um preço a ser pago pela simplicidade e facilidade de implementação da técnica. Para melhor caracterizar a complexidade algorítmica nos cálculos do controlador foi medido o tempo de processamento, ou o esforço computacional na derivação dos parâmetros do controlador. O GPC padrão consome cerca de $5.3 \times 10^{-3}$ s e o CPOM aproximadamente $1.2 \times 10^{-5} \mathrm{~s}$, assim, o CPOM é cerca de 440 vezes mais rápido que o algoritmo do GPC padrão.
Como continuação deste trabalho pretende-se analisar, implementar e avaliar a abordagem da técnica para modelo interno de segunda ordem com atraso de transporte (SOPDT), proposta por Neshasteriz et al., 2010.

\section{Agradecimentos}

Agradecemos ao Departamento de Automação e Sistemas da UFSC, GPqTCA, WEG Automação e a CAPES pelo apoio na realização deste trabalho.

\section{Referências Bibliográficas}

Besch, G. V. L.; Vale, M. R. B. G.; Silva, H. A.; Araujo, F. M. U. and Maitelli, A. L (2009). Implementação de um controlador GPC escalonado em um CLP. V Congresso Rio Automação.

Camacho, E. F. and Bordons, C (1998). A Generalized Predictive Controller for a Wide Class of Industrial Processes. IEEE Transaction on Control Systems Technology, Vol.6, No.3, pp. 372-387. DOI: $10.1109 / 87.668038$

Camacho, E. F. and Bordons, C (2007). Model Predictive Control. Springer-Verlag, London. DOI: 10.1007/978-0-85729-398-5

Coelho, A. A. R. and Coelho, L. S. (2004). Identificação de Sistemas Dinâmicos Lineares. Editora da UFSC, Florianópolis - SC.

Clarke, D. W.; Mohtadi, C. and Tuffs, P. S (1987). Generalized Predictive Control - Part 1. The Basic Algorithm. Automatica, Vol.23, No.2, pp. 137-148. DOI: 10.1016/0005-1098(87)90088-4

Cooper, D. J. (2004). Pratical Process Control Using Control Station. Control Station LLC, Storrs.

Haber, R; Bars, R. and Schmitz U. (2011). Predictive Control in Process Engineering. Wiley \& Sons.

Lopes Junior, C. A. A. (2013). Síntese de Algoritmos de Controle Preditivo em PLC300-WEG com Aplicação em Plantas em Escala de Laboratório e na Regulação da Velocidade de Turbinas Hidráulicas. Dissertação (Mestrado), Departamento de Automação e Sistemas, Universidade Federal de Santa Catarina.

Rohman, A. S. and Sirius, R. (2011). Model Predictive Control Implementation on a Programmable Logic Controller. International Conference on Electrical Engineering and Informatics, pp. 1-4.

Palomo, G. V. and Rossiter J. (2011). Efficient Suboptimal Parametrics Solutions to Predictive Control for PLC Applications. Control Engineering Pratice, Vol.19, No. 7, pp. 732-743. DOI: 10.1016/j.conengprac.2011.04.001

Moradi, M.; Katebi, M. and Johnson, M. (2001). Predictive PID Control: A New Algorithm. The 27th Annual Conference of the IEEE.

Neshateriz, A. R.; Sedigh, A. K. and Sadjadian, H. (2010). Generalized Predictive Control and Tuning of Industrial Processes with SOPDT Models. Journal of Process Control. Vol.20, pp.63-72 DOI: 10.1016/j.jprocont.2009.10.003

Seborg, D. E.; Mellichamp, D. A. and Eggar $20_{1}$ TSBMAC $_{\text {SBMAC }}$ (2010). Process Dynamics. Wiley and Sons. 